\title{
Review on Hot Melt Extrusion Technology and it's Application
}

\author{
Mali A. S*., Gavali K.V., Choudhari R. G., Anekar V.P., Gavhane Y.N.
}

Department of Pharmaceutics, Government College of Pharmacy Karad, Tal-Karad, Dist- Satara, Maharashtra, India

\section{ABSTRACT}

The use of hot-melt extrusion (HME) within the pharmaceutical industry is steadily increasing, due to proven its ability to efficiently manufacture novel products. With the use of HME we can increase solubility and bioavailability of the API. HME has proven to be a robust method of producing numerous drug delivery systems like modified, controlled and targeted drug delivery systems. resulting improved bioavailability as well as taste masking of bitter active pharmaceutical ingredients (APIs). Hot-melt extrusion techniques are pragmatic in the manufacture of a variety of dosage forms and formulations such as granules, pellets, tablets, suppositories, implants, stents, transdermal systems and ophthalmic inserts. In This paper we review about the types of Extruder, solid dispersion and Application of the HME to the pharmaceutical industries.

Keywords : Hot-Melt Extrusion, Targeted Drug Delivery, Bioavailability.

\section{INTRODUCTION}

Industrial applications of hot melt extrusion date back to the early 1900s. This process has since grown in use, and today is one of the most widely applied unit operations in the polymer industry. Recent increases in both patents and publications indicate that it is rapidly becoming a key processing route for pharmaceutical dosage units as well (1).

Hot-melt extrusion (HME) is a processing technique widely used to convert plastic raw materials into a product of uniform shape and density by heating and forcing them through a die, generally employing a rotating screw. Despite some limitations implied by the thermal stability of drugs and formulation aids as well as the need for thermoplastic carriers (2)

Hot melt extrusion (HME) is the process of applying heat and pressure to melt a polymer and forcing it through an orifice in a continuous process.
HME is well-known, developed to produce polymer products of uniform shape and density.To date HME has emerged as a novel processing technology in developing molecular dispersions of active pharmaceutical ingredients (APIs) into various polymer and lipid matrices which has led this technique to demonstrate time controlled, modified, extended, and targeted drug delivery (3-6).

This makes HME an excellent alternative to other conventionally available techniques such as roll spinning and spray drying. In addition to being a proven manufacturing process, HME meets the goal of the US Food and Drug Administration's (FDA) process analytical technology (PAT) scheme for designing, analysing, and controlling the manufacturing process via quality control measurements during active extrusion process (6 -7)In this chapter, the hot-melt extrusion technique is reviewed based on a holistic perspective of its various components, processing technologies, and the 
materials and novel formulation design and developments in its varied applications in oral drug delivery systems.

\section{HISTORY:}

Extrusion is a well-known processing technology that has been developed during the last century. It has been used in many diverse industrial fields, mostly with the processing of foods and the manufacturing of plastics. The first industrial use of single-screw extruders was in the early 1930s with the extrusion of thermoplastic materials(8).However, an early singlescrew extruder was designed by Sturges in 1871 for the purpose of pumping soap(9).Early twin-screw extruders were attributed to Wiegardin in 1874 and $\mathrm{P}$ fleiderer in 1881(11).The first commercially available twin-screw extruders came to the market in the 1940s. Both single-screw and twin-screw extruders were initially developed within a similar time frame, at the end of the 1800s, but the commercialization and the widespread use of single-screw system occurred earlier than that of twin-screw systems. This can be explained by several engineering issues for twinscrew extruders that were not overcome until the 1940s. (10)

\section{METHODS AND MATERIAL}

\section{PROCESS TECHNOLOGY OF HOT- MELTEXTRUSION:}

Hot-melt extrusion equipment consists of an extruder, auxiliary equipment for the extruder, downstream processing equipment, and other monitoring tools used for performance and product quality evaluation (12). The extruder is typically composed of a feeding hopper, barrels, single or twin screws, and the die and screw-driving unit the auxiliary equipment for the extruder mainly consists of a heating/cooling device for the barrels, a conveyer belt to cool down the product and a solvent delivery pump. The monitoring devices on the equipment include temperature gauges, a screw-speed controller, an extrusion torque monitor and pressure gauges. (13) The theoretical approach to understanding the melt extrusion process is therefore, generally presented by dividing the process of flow into four sections:

1) feeding of the extruder through a hopper,

2) mixing, grinding, reducing the particle size, venting, and kneading,

3) flow through the die, and

4) extrusion from the die and further downstream processing.

Generally, the extruder consists of one or two rotating screw inside a stationary cylindrical barrel. The barrel is often manufactured in sections, which are bolted or clamped together. An end-plate die, connected to the end of the barrel, determines the shape of the extruded product. (10)

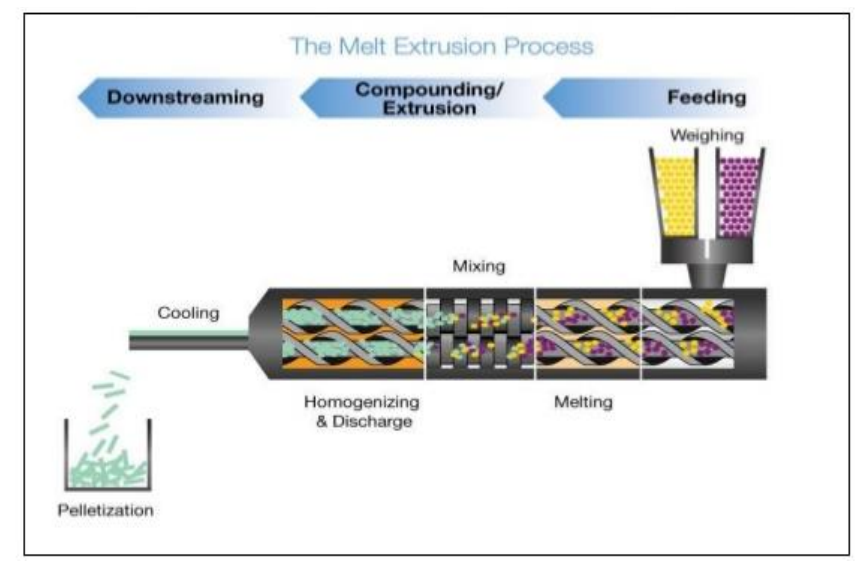

Figure 1: hot melt extrusion process

\section{Single-Screw and Twin-Screw Extruder:}

(A) Single Screw Extruder: The single-screw extruder has been the most widely used. Twin-screw extruders use two side-by-side screws either co-rotating or counter-rotating. There are several advantages of twin-screw extruders over single-screw extruders such as easier material feeding and dispersion 
capacities, less tendency to over-heat and shorter transit times. However, single-screw extruders are more simple and cheaper. The single screw extruder is the most widely used extrusion system in the world. One screw rotates inside the barrel and is used for feeding, melting, devolatilizing, and pumping. Mixing is also accomplished for less demanding applications. Single screw extruders canbe either flood or starve fed, depending upon the intended manufacturing process. Single screw extruders are continuous, high-pressure pumps for viscous materials that can generate thousands of pounds of pressure while melting and mixing. (14) Most extruder screws are driven from the hopper end. However, once screws are reduced to less than $18 \mathrm{~mm}$, the screw becomes weak and solids transportation is far less reliable [Fig 2].

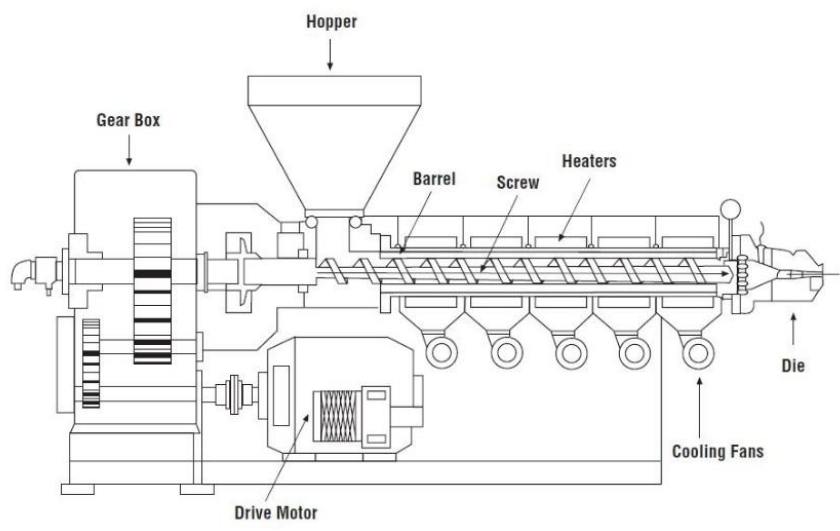

Figure 2: Single screw extruder

(B) Twin-screw extruder: The twin-screw extruder is characterized by the following descriptive features:

1. Short residence time: The residence time in the twin-screw extruder in a typical extrusion processes ranges from 5-10 minutes depending on the feed rate and screw speed.

2. Self-wiping screw profile: The self-wiping screw profile i.e. the flight of the one Screw wipes the root of the screw on the shaft next to it, ensures near complete emptying of the equipment and minimizes product wastage on shutdown.

3. Minimum inventory: Continuous operation of the equipment coupled with the continuous feeding of the material helps in reducing inventories of work in progress. This is important when processing valuable or potentially hazardous materials.

4. Versatility: Operating parameters can be changed easily and continuously to change extrusion rate or mixing action (14) [Fig 3].

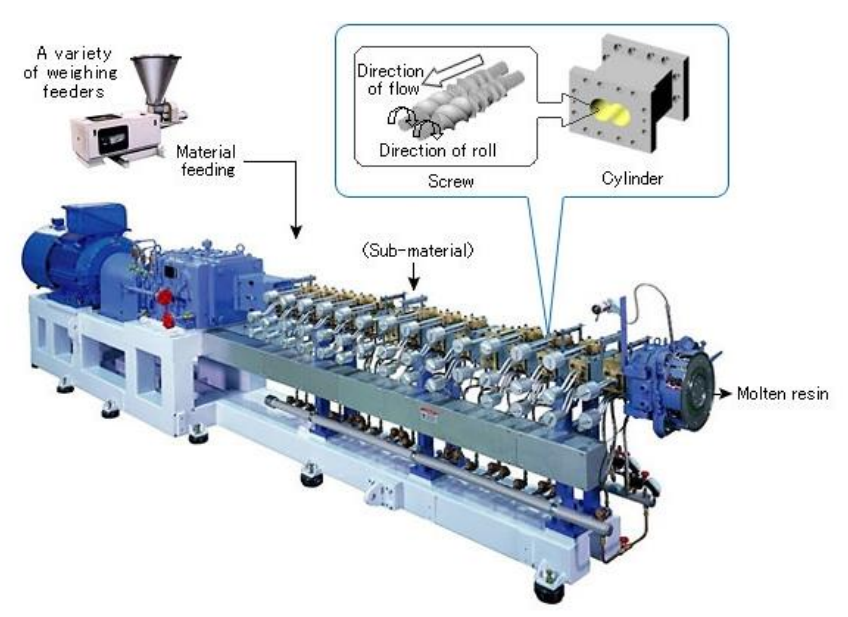

Figure 3 twin screw extruder.

\section{ADVANTAGES AND DISADVANTAGES OF HME}

HME offers several advantages over conventionally available pharmaceutical processing techniques including

(a)increased solubility and bioavailability of water insoluble compounds.

(b) solvent-free nonambient process

(c) economical process with reduced production time, fewer processing steps, and a continuous operation.

(d) capabilities of sustained, modified, and targeted release.

(e) better content uniformity in extrudates.

(f) no requirements for the compressibility of active ingredients.

(g) uniform dispersion of fine particles.

(h) good stability at changing $\mathrm{pH}$ and moisture levels and safe application in humans.

(i) reduced number of unit operations and production of a wide range of performance dosage forms.

(j) a range of screw geometries. (15-18) 
However, HMEhas some disadvantages as well. The main drawbacks of HME include thermal process (drug/polymer stability), use of a limited number of polymers, high flow properties of polymers, and excipients required and not suitable for relatively high heat sensitive molecules such amicrobial species and proteins (17-18).

\section{EVALUATION OF HME FORMULATIONS}

The extrudes obtained from the extruder can be evaluated by using a number of techniques. These methods are also useful to differentiate between solid solutions (molecularly dispersed drugs) and solid dispersions (physical mixtures of drug and carrier).

1.Differential scanning calorimetry (DSC): It can be used for the quantitative detection of transitions like $\mathrm{Tg}$ and $\mathrm{Tm}$ in which energy is required or liberated. It is also used for the study of drug excipient incompatibility and to identify amorphous and crystalline forms in extrudes.

2.Thermo gravimetric analysis (TGA): TGA can be used as a screening tool for the thermal stability of materials used in HME. TGA is limited to studies involving either a weight gain or loss and is commonly used to study desolvation and decomposition. TGA is a measure of thermally induced weight loss of a material as a function of applied temperature.

3.X-Ray diffraction pattern (XRD): XRD is also used to characterize the crystalline properties of hot-melt extruded dosage forms. Crystallinity is reflected by a characteristic fingerprint region in the diffraction pattern. However, the sensitivity of the XRD technique is limited and cannot generally detect crystallinity of less than $10 \%$.

4.Infrared spectroscopy (IR): Infrared spectroscopy can be used to differentiate between peaks that are sensitive to changes in crystallinity.

5.Nuclear Magnetic Resonance (NMR): Solid state nuclear magnetic resonance (NMR) has been used to probe the crystallinity of materials and to detect changes in bonding between functional group.

6.Microscopy: Microscopy is one of the best methods to study the crystalline properties of hot-melt extrudates. Both optical and electron methods are suitable to examine the surface morphology of samples to probe for the presence of crystalline particles or amorphous domains. It is also possible to obtain reliable particle size information using these techniques.

7.In- vitrodissolution testing: Determination of release profile to surrogate in-vivo performance.

8.Mechanical analysis: Tensile strength and elongation has to be checked to know the plasticity and brittleness of extrudes (19).

\section{APPLICATIONS OF HOT MELT EXTRUSION:}

\section{A. Overall Applications of HME}

Extrusion technology is one of the most important fabrication processes in the plastic and rubber industries. Products made from melt extruded polymers range from pipes to hoses through to the insulated wires, cables, rubber sheeting, and polystyrene tiles. Plastics that are commonly processed by HME technique include acrylics and cellulosic, polyethylene, poly propylene, polystyrene, and vinyl plastics(20-21). In the food industry, extrusion has been utilized for pasta production with a widely used multitalented technique combining cooking and extrusion in a self-styled extrusion cooker (22). In the animal feed industry and veterinary science, extrusion is commonly applied as a means of producing pelletized feeds, implants, or injection moulding (23). HME has successfully been applied in the formulation of fast dispersing PVP melt extrudates of poorly soluble active agents as solid molecular dispersions in the crop protection field (24). HME technology has already achieved a strong place in the pharmaceutical industry and academia due to several advantages over traditional processing methods such as roll spinning and grinding. In 
addition to being an efficientMfg process, HME enhances the quality and efficacy of manufactured products and therefore over the past few years HME has emerged as a novel technique in pharmaceutical applications.

The main use of HMEis to disperse active pharmaceutical ingredients (APIs) in a matrix at the molecular level, thus forming solid solutions (25). In the pharmaceutical industry, HME has been used for various applications, such as (i) enhancing the dissolution rate and bioavailability of poorly soluble drugs by forming a solid dispersion or solid solution,

(ii) controlling or modifying the release of the drug, (iii) taste masking of bitter APIs, and (iv) formulation of various thin films The bioavailability of an active ingredient is controlled byits aqueous solubility. Therefore, increasing the solubility of water insoluble drugs is still a real challenge in the formulation development process (7).

Due to the advent of high throughput screening (HTS) in the drug discovery process, the resultant compounds are often high molecular weight and highly lipophilic and therefore exhibit poor solubility [29]. Scientists have already tried to address solubility issues by various pharmaceutical interventions. Among the many methods available to improve solubility and dissolution rate, preparation of solid dispersions and solid solutions has gained vast attention. For that reason, HME has been successfully applied to prepare solid molecular dispersion of APIs into different hydrophilic polymer matrices (25-26).

\section{B. HME IN SOLID DISPERSIONS:}

Due to high screening in drug discovery, up to $50 \%$ of the discovered new drug candidates have very poor solubility and therefore low bioavailability. Improvement of solubility, dissolution rate and absorption of drugs with low water-solubility are challenging aspects in the development of pharmaceutical products. Solid dispersions are an approach to increase the solubility of the API and increase bioavailability, which is partly explained by a reduction in particle size [27]. Classification of solid dispersions can be divided into molecular or particulate dispersions, based on the drug solubility in the carrier.

If the drug is dispersed at the molecular level, the terms molecular dispersion or solid solution are used. However, if the drug is dispersed at the particulate level, the terms particulate dispersion or solid suspension are used. In a molecular solid dispersion, the active ingredient is molecularly embedded in an inert carrier. A true solid solution can be formed if complete miscibility between the components is achieved, leading to the API being molecularly dispersed throughout the polymer.

The drug exists in a thermodynamically unstable amorphous form. Factors to consider when forming solid solutions are the solid-state solubility of the API in the carrier, the interaction between them and the stability of the formulation to form a particulate dispersion, the drug should have limited solubility in the polymer carrier. The polymer/drug ratio can be much less compared to molecular dispersions due to the lower number of polymers necessary to coat the particles.

The polymer intermixes itself between adjacent drug crystals leading to a more thermodynamically stable crystalline form. Although particulate dispersions are more stable than molecular dispersion, the improved dissolution rate and absorption might not be as large [11]. One of the advantages with hotmelt extrusion is that it can disperse drugs in a matrix down to the molecular level, forming a solid solution. At elevated temperatures in the extruder, the solubility of the drug in the polymer carrier is increased, resulting in the formation of a solid solution if the compounds are miscible.

Depending on the processing conditions and miscibility of the components, a solid particulate 
dispersion might also be formed where the drug is only partly dispersed and physical mixture of drug and carrier exist. Solid dispersions have been described to increase dissolution, absorption and therapeutic efficacy of drugs. However, there are very few products on the pharmaceutical market that are solid dispersion systems [11]. Problems that limit the commercial application of solid dispersions are the method of preparation, reproducibility, formulation into dosage forms and scale up. All of these problems might be overcome by hot-melt extrusion.

\section{Hot-melt extrusion in the pharmaceutical industry}

The medical device industry has been using HME to manufacture medical devices such as catheters and tubing for many years. Within the pharmaceutical arena, HME was the obvious production method since a number of the products required for medical purposes are cylindrical and transferred with relative ease from plastic tube production utilized for decades within the plastics industry (28). More recently, new engineering techniques have been utilized as a means of making HME a viable processing technique for drug-delivery purposes. This is certainly reflected in the steady increase in the number of drug-delivery patents incorporating this technology (27).

One of the key drivers for the adoption of HME within the pharmaceutical industry is advantages associated to increased throughput, efficient mixing and material modification during the extrusion process. Moreover, HME is a highly versatile technology capable of producing a wide range of different drug-delivery products including pellets, controlled-release tablets, oral fast-dissolving systems, transdermal/transmucosal delivery systems and implants (10).

\section{FUTURE TREND:}

Today melt extrusion technology represents an efficient pathway for manufacture of drug delivery systems. The application of HME technology in the pharmaceutical industry has tended to focus on the development of bio-enhanced formulations to increase the efficacy of poorly water-soluble compounds. There has also been an increase in the application of HME for the development of controlled release formulations.

\section{Conclusion:}

Hot melt extrusion is emerging as a leading technology for increasing drug solubility and enhancing bioavailability. The potential of the technology is reflected in the wide scope of different dosage forms including oral dosage forms, implants, bio adhesive ophthalmic inserts, topical films, and effervescent tablets. It has also been documented that HME is a solvent-free, robust, quick and economy favoured manufacturing process for the production of a large variety of pharmaceutical dosage forms.

\section{REFERENCES}

[1]. Repka, M., Majumdar S., Battu, S.K. et al.."Application of hot-melt extrusion for drug delivery,"Expert Opin. Drug.Deliv. 52012 p no. 1357-1376 (2008).

[2]. Giulia L., Alessandra M., et al. "Evaluation of hot-melt extrusion technique in the preparation of HPC,'European Journal of Pharmaceutical Sciences.p no.77-85 (2014).

[3]. M.Maniruzzaman, J. S. Boateng, M. Bonnefille, A. Aranyos, J. C. Mitchell, and D. Douroumis, "Taste masking of paracetamol by hot melt extrusion: an in vitro and in vivo evaluation," European Journal of Pharmaceutics and Biopharmaceutics, vol. 80, no. 2, p no. 433-442, (2012).

[4]. M. A. Repka, S. Shah, J. Lu et al., "Melt extrusion: process to product," Expert Opinion on Drug Delivery, vol. 9, no. 1, pp. 105-125, (2012). 
[5]. M. A. Repka, S.Majumdar, S. K. Battu, R. Srirangam, and S. B. Upadhye, "Applications of hot-melt extrusion for drug delivery," Expert Opinion on Drug Delivery, vol. 5, no. 12, pp. 1357- 1376, (2008).

[6]. M. A. Repka, S. K. Battu, S. B. Upadhye et al., "Pharmaceutical applications of hot-melt extrusion: part II," Drug Development and Industrial Pharmacy, vol. 33, no. 10, pp. 10431057, (2007).

[7]. Mohammed Maniruzzaman, Joshua S. Boateng, Martin J. Snowden, 1 and Dennis Douroumis, "A Review of Hot-Melt Extrusion: Process," Technology to Pharmaceutical Products International Scholarly Research Network page no 1 to 9 (2012).

[8]. Sturges, J.D. Improvement in apparatus for cooling and mixing soap. US Patent 114063,(1877).

[9]. RinaChokshi, Hossein Zia,"Hot-Melt Extrusion Technique: A Review," Iranian Journal of Pharmaceutical Research 3 to 16(2004).

[10]. Ankitpatel, Deepak Sahu, Ashok Dashora, "A Review of Hot Melt Extrusion Technique International Journal of Innovative Research in Science, Engineering and Technology,"Vol. 2, p no. 2194 to 2198 (2014).

[11]. Pfleiderer, P., "Innovations on kneading and mixing machines of Freyburger type," German Patent 18797, (1882).

[12]. Kruder GA.,"Extrusion. In: Encyclopedia of Polymer Science and Engineering"Vol. 1, 2nd ed. John Wiley \& Sons Inc., New York 571631(1985).

[13]. Breitenbach J.,"Melt extrusion: from process to drug delivery technology,"Eur. J. Pharm. Biopharm. p no.107-117 (2002).

[14]. SandhyaPamu ,C. V. S. Subrahmanyam and K. S. K. RaoPatnaik Hot melt extrusion technique and its pharmaceutical applications: A review international journal of pharmacy and analytical research Vol.3 524 to 530 (2014).

[15]. J.W.McGnity and J. J. KOleng, "Preparation and evaluation of rapid release granules using novel melt extrusion technique," American Association of Pharmaceutical Scientists, $\mathrm{p}$ no. 153- 154, (2004).

[16]. D. S. Jones, "Engineering drug delivery using polymer extrusion/ injection moulding technologies," School of Pharmacy, vol. 4-9, pp. 18-27, (2008).

[17]. H. H. Grunhagen and O. Muller, "Melt extrusion technology," Pharmaceutical Manufacturing International, vol. 1, pp. 167170, (1995).

[18]. S. Singhal, V. K. Lohar, and V. Arora, "Hot-melt extrusion technique," WebmedCentral Pharmaceutical Sciences, vol. 2, no. 1, Article ID 001459, 2011.

[19]. DevendraRidhurkar, Attila Vajdai1 and ZsoltZsigmond, "Hot-melt extrusion (HME)and its application for pharmacoki $\neg$ netic improvement of poorly water soluble drugs,"Journal in the field ofPharmacology, Toxicology andBiomedical Reports

[20]. J. Breitenbach, "Melt extrusion: from process to drug delivery technology," European Journal of Pharmaceutics and Biopharmaceutics, vol. 54, no. 2, p no. 107-117, (2002).

[21]. M.Mollan, "Historical overview in Pharmaceutical Extrusion Technology," I. Ghebre-Sellassie and C. Martin, Eds., p no. 118, CRC Press, New York, NY, USA, (2003).

[22]. A. Senouci, A. Smith, and P. Richmond, "Extrusion cooking," Chemical Engineer, no. 417, p no. 30-33, (1985).

[23]. E. Sebestyen, "Problems of grains preservation in storage facilities," Journal of Flour and Animal FeedMilling, vol. 10, p no. 24- 25, (1974). 
[24]. D. J. Wedlock and D. V. Wijngaarden, "Fast dispersing solid PVP-containing crop protection formulation and process therefore," US Patent 1992, 5: 665, 369.

[25]. M.Maniruzzaman, M. Rana, J. S. Boateng, and D. Douroumis, "Dissolution enhancement of poorly water-soluble APIs processed by hotmelt extrusion using hydrophilic polymers," Drug development and Industrial Pharmacy. $\mathrm{p}$ no 1254 to 1274 (2012)

[26]. X. Zheng, R. Yang, X. Tang, and L. Zheng, "Part I: characterization of solid dispersions of nimodipine prepared by hotmelt extrusion," Drug Development and Industrial Pharmacy, vol. 33, no. 7, p no. 791-802, (2007).

[27]. Mollan M., Historical Overview in Pharmaceutical Extrusion Technology, edsGhebre-Sellasie, I. \& Martin C. (Marcel Dekker, Inc., New York), p no 1-18,(2003).

[28]. Matthew W., Marcia w., David J. \& Gavin A.,"Hot-melt extrusion technology and pharmaceutical application," future science $\mathrm{p}$ no. 786 to 797 (2012).

\section{Cite this article as :}

Mali A. S., Gavali K. V., Choudhari R. G., Anekar V. P., Gavhane Y. N., "Review on Hot Melt Extrusion Technology and it's Application", International Journal of Scientific Research in Science and Technology (IJSRST), Online ISSN : 2395-602X, Print ISSN : 2395-6011, Volume 6 Issue 6, pp. 253-260, November-December 2019. Available at doi : https://doi.org/10.32628/IJSRST196653 Journal URL : http://ijsrst.com/IJSRST196653 